\title{
Alocação de Taxa de Transmissão Utilizando Predição do Tráfego de Rede Baseada no Expoente de Lyapunov
}

\author{
Évelynn R. Rosa , Flávio G. C. Rocha \\ ${ }^{1}$ Escola de Engenharia Elétrica, Mecânica e Computação. \\ Universidade Federal de Goiás, Goiânia-GO, Brasil. \\ wltnschng@gmailcom, flaviogcreufg.br
}

\begin{abstract}
This paper evaluates the performance of algorithms developed with the purpose of quantifying chaos theory parameters. Initially, a standard procedure is described, within the spectrum of Chaos Theory and its most notable procedures, in order to analyse and classify experimental data which displays non-linear characteristics. A method is then introduced, to predict the traffic and propose a dynamic rate allocation for network servers based on the Lyapunov exponent and the Hurst parameter. Reconstructing the attractor, determining embedding delay and correlation dimension, estimating the Lyapunov exponent and the Hurst parameter, predicting the traffic, and performing rate allocation for the network servers are the steps followed in this analysis.
\end{abstract}

Resumo. Esse artigo avalia o desempenho de algoritmos desenvolvidos com o propósito de quantificar parâmetros referentes à teoria do caos. Inicialmente, um procedimento padrão é descrito, dentro do espectro da Teoria do Caos e seus procedimentos mais notáveis, para a análise e medida de dados experimentais que apresentam características não-lineares. Um método é então proposto, para a predição de tráfego e a alocação dinâmica de taxa de transmissão para servidores de rede, baseados no expoente de Lyapunov e no parâmetro de Hurst. Reconstruir o atrator, determinar o atraso ideal e dimensão de correlação, estimar o expoente de Lyapunov e o parâmetro de Hurst, prever o tráfego, e alocar a taxa de transmissão correspondente são os passos seguidos nessa análise.

\section{Introdução}

A teoria do caos descreve uma variedade de sistemas não-lineares e por isso já foi abordada nas mais diversas áreas como a meteorologia ([Lorenz 1995]), a química e filosofia ([Prigogine and Stengers 1984]), o jornalismo ([Gleick 1987]), física ([Strogatz 2012]), a matemática ([Mandelbrot and Hudson 2004]), a construção de reservatórios ([Hurst 1956]), a previsão do tempo ([Lorenz 1963]), os ciclos pluviais e a bolsa de valores ([Mandelbrot 1968], [Mandelbrot 1997], [Alves et al. 2018], [Alves 2019]), a topologia ([Tempelman and Khasawneh 2020]), e a coleta e processamento de dados ([Trinh and Miki 1999], [Feng et al. 2009], [Rocha and Teles 2013], [Liu and Zhang 2016], [Alves et al. 2017], [Tian 2020]).

O comportamento das redes de dados de forma geral apresenta características que possibilitam sua análise de acordo com essa abordagem não-linear adotada pela teoria do caos, de forma que possam ser analisadas através de alguns parâmetros específicos como a dimensão de correlação, o expoente de 
Lyapunov e o parâmetro de Hurst, como já foi discutido em inúmeros trabalhos clássicos ([Takens 1981], [Mañé 1981], [Grassberger and Procaccia 1983b], [Liebert and Schuster 1989], [Kaplan and Glass 1993]). Nestes ambientes, o interesse consiste em capturar características fundamentais de comportamento do sistema a fim de garantir a qualidade de serviço ( $Q o S$ - Quality of Service) através do monitoramento de métricas tais como banda, atraso, probabilidade de perda de pacotes e taxa de alocação [Solis 2007], [Feng et al. 2009], [Rocha 2016], e da análise de características comportamentais dos dados por meio de grandezas como o expoente de Lyapunov e o parâmetro de Hurst, por exemplo.

Para esse propósito, este trabalho classifica, através desses parâmetros, a natureza dos dados analisados, medindo seu comportamento ao longo do tempo a fim de prever seu comportamento futuro, considerando suas características intrínsicas de dependência de longa duração, quantificadas pelo parâmetro de Hurst, e de dimensionalidade, determinada pela dimensão de correlação. Esta análise é possibilitada, neste trabalho, principalmente pela reconstrução do espaço de fase [Wolf et al. 1985], [Rosenstein et al. 1993].

O restante deste estudo está organizado da seguinte maneira: na Seção 2 descrevem-se as três abordagens de análise: o método da autocorrelação, o expoente de Lyapunov e o parâmetro de Hurst. Os experimentos realizados nas séries temporais estão descritos na Seção 5, enquanto na Seção 6 estão as conclusões.

\section{Medidas de Classificação do Tráfego com Base na Teoria do Caos}

O primeiro passo deste estudo do comportamento caótico de um sistema é a reconstrução do espaço de fase, descrito à seguir na Seção 2.1.

\subsection{Reconstrução do Espaço de Fase}

A reconstrução do espaço de fase a partir dos dados medidos é um procedimento inicial padrão na análise de séries temporais não-lineares, durante o qual toma-se um espaço original multidimensional com o intuito de projetá-lo em um espaço reconstruído equivalente de dimensionalidade mais baixa. Para tal propósito, define-se um vetor bidimensional

$$
\mathbf{x}(t)=(\mathbf{B}(t), \mathbf{B}(t+\tau)),
$$

para um atraso $\tau>0$ (Seção 2.2), de forma que a série temporal $\mathbf{B}(t)$ gere uma trajetória $\mathbf{x}(t)$ em um espaço de fase bidimensional. Ao definir-se um vetor tridimensional

$$
\mathbf{x}(t)=(\mathbf{B}(t), \mathbf{B}(t+\tau), \mathbf{B}(t+2 \tau)),
$$

obtém-se um espaço de fase tridimensional, e assim sucessivamente.

Uma série temporal representa, então, uma sequência escalar de medidas de alguma quantidade que reflete o estado do sistema no instante de medida, coletadas em múltiplos de uma amostragem temporal fixa:

$$
x_{n}=x(\mathbf{s}(n \Delta t))+\eta_{n},
$$

na qual s é a função de medida e $\eta_{n}$ é o ruído inerente à medida. Uma reconstrução por atrasos em $m$ dimensões, desconsiderando o ruído $\eta_{n}$, é então constituída pelos vetores $\mathbf{X}_{n}$, construídos da seguinte maneira:

$$
\mathbf{X}_{n}=\left(x_{i}, x_{i+\tau}, \ldots, x_{i+(m-1) \tau}\right),
$$


na qual $\tau$ é o atraso de reconstrução, $m$ é a dimensão de incorporação, e $\mathbf{X}_{n}$ é o vetor $m$-dimensional. A trajetória reconstruída $\mathbf{X}$ pode então ser expressa como uma matriz na qual cada linha $\mathbf{X}_{n}$ é um vetor estado de fase de $m$ atrasos, ou seja:

$$
\boldsymbol{X}=\left(\boldsymbol{X}_{1}, \boldsymbol{X}_{2}, \ldots, \boldsymbol{X}_{N}\right)^{T}
$$

o intervalo temporal entre as amostras é o atraso $\tau$, e o atrator formado por $\mathbf{X}_{n}$ será equivalente ao atrator no espaço desconhecido ao qual o sistema original pertence se a dimensão $m$ do espaço de coordenadas de atraso for suficientemente alta.

$\mathbf{X}$ será então uma matriz $N \mathrm{x} m$, e as constantes $N, n, m$ e $\tau$ se relacionam da seguinte maneira:

$$
N=n-(m-1) \tau
$$

Portanto, a reconstrução do espaço de fase requer o cálculo de variáveis que determinam sua estrutura (Equação 6), como a dimensão de incorporação $m$, tratada a seguir. Ela é escolhida de forma que $m>2 D_{2}+1$, na qual $D_{2}$ é a dimensão de correlação (Seção 2.3). Se todos esses requisitos forem cumpridos, então o espaço de fase reconstruído é equivalente ao original [Takens 1981], [Mañé 1981].

\subsection{O atraso $\tau$}

A distância espacial entre as posições das medidas é denominada de atraso $\tau$, como mostram as Equações 1, 2, e 4, e seu valor apropriado é usado na reconstrução do espaço de fase, havendo, portanto, na escolha desse valor um dilema entre redundância e decorrelação entre as amostras da série temporal: a meta é diminuir a redundância enquanto se preserva a correlação uma vez que quanto menor a distância entre as amostras mais elas tendem estar correlacionadas. Por outro lado, elas podem parecer completamente não-correlacionadas se estiverem muito distantes entre si [Kantz and Schreiber 2004].

Um método clássico de cálculo do atraso ideal $\tau$ é o da autocorrelação, segundo o qual esse valor é obtido de forma que a função de autocorrelação atinja o valor de $1-1 / e,(e=2.7183)$ [Rosenstein et al. 1993], [Feng et al. 2009].

\subsection{Dimensão de Correlação}

A dimensão de correlação, inicialmente descrita em [Grassberger and Procaccia 1983a] e [Grassberger and Procaccia 1983b], define como o objeto geométrico de interesse deve ser reconstruído a partir de uma amostra finita de dados pontuais. O método define a dimensão de correlação $D_{2}$ por meio do seguinte cálculo:

$$
D_{2}=\lim _{r \rightarrow 0} \frac{\partial \ln C_{m}(r)}{\partial \ln (r)},
$$

na qual $C_{m}(r)$ é a integral de correlação que deve ser calculada da seguinte maneira:

$$
C(r) \approx \frac{2}{N(N-1)} \sum_{i=1}^{N} \sum_{j=i+1}^{N} \Theta\left(r-\left\|\mathbf{x}_{i}-\mathbf{x}_{j}\right\|\right),
$$


na qual $\Theta$ é a função degrau de Heaviside, que estipula que $\Theta(x)=0$ se $x \leq 0$ e $\Theta(x)=1$ se $x>0$. O somatório conta os pares $\left(\mathbf{x}_{i}, \mathbf{x}_{j}\right)$ cuja distância entre si é menor que $r$, e $\left\|\mathbf{x}_{i}-\mathbf{x}_{j}\right\|$ é a distância entre os vetores $Y_{i}$ e $Y_{j}$, definida como:

$$
\left\|\mathbf{x}_{i}-\mathbf{x}_{j}\right\|=\max \left\{\left|x_{i+l \tau}-x_{j+l \tau}\right|: 0 \leq l \leq m-1\right\}
$$

$D_{2}$ é , então, a inclinação da reta:

$$
D_{2}=\frac{d \ln C_{m}(r)}{d \ln (r)}
$$

Ou seja, uma vez que os vetores de incorporação $\mathbf{X}_{n}$ são reconstruídos, a estimativa da dimensão de correlação poderá ser feita em dois passos: primeiramente deve-se calcular o somatório da correlação definida pela Equação 8, na faixa estipulada para $r$ e para várias dimensões de incorporação $m$ (Figura 3), para então se inspecionar $C(r)$ a fim de verificar a presença de auto-similaridade: se for satisfatória, pode-se subsequentemente calcular um valor para a dimensão $D_{2}$ [Kantz and Schreiber 2004].

\subsection{Dimensão de Incorporação - $m$}

A última variável da Equação 6, cujos valores numéricos são usados na reconstrução do espaço de fase, é a dimensão de incorporação $m$, a qual diz respeito à quantidade de vetores atraso a serem reconstruídos na matriz de reconstrução do espaço de fase [Kantz and Schreiber 2004], [Strogatz 2015].

Tecnicamente, é preciso que existam tantos atrasos (posições no vetor atraso, Equação 4) quanto necessários para que o atrator subjacente possa ser nitidamente visualizado no espaço de fase, e por isso o procedimento usual é aumentar a dimensão de incorporação $m$ a cada iteração e assim calcular as dimensões de correlação $D_{2}$ dos atratores resultantes (Figura 3). Os valores aumentarão sucessivamente até que a dimensão de incorporação atinja um valor alto o bastante (Figura 4), permitindo assim que o atrator e o valor estimado da dimensão de correlação $D_{2}$ se estabilizem em um valor "verdadeiro" [Strogatz 2015].

Uma vez que o espaço de fase tenha sido reconstruído é possível medir o grau de expansão das órbitas do atrator através do expoente de Lyapunov, descrito na Seção 2.5, e de dependência entre as amostras através do parâmetro de Hurst, descrito na Seção 2.6. Em posse desses quantificadores é possível realizar a previsão do tráfego, procedimentos estes que serão descritos na Seção 3.

\subsection{O Expoente de Lyapunov}

Nos sistemas periódicos as trajetórias predominantemente divergem lentamente ao longo do tempo, mas nos sistemas caóticos essa separação é exponencialmente rápida. $\mathrm{O}$ valor médio desse aumento, o expoente de Lyapunov, é característico de cada sistema, e quantifica a intensidade do caos ao caracterizar a divergência das trajetórias à medida que o fluxo caótico flui [Kantz and Schreiber 2004], [Berge et al. 1987].

Dois são os algoritmos clássicos de cálculo do expoente de Lyapunov, descritos em [Wolf et al. 1985] e [Rosenstein et al. 1993]. O primeiro é amplamente usado mas susceptível a resultados errôneos, uma vez que ele não testa a presença de divergência 
exponencial, e apenas assume que ela esteja presente. $\mathrm{O}$ segundo testa diretamente a presença da divergência de trajetórias adjacentes permitindo assim que se decida se faz sentido calcular o expoente de Lyapunov para um determinado conjunto de dados [Kantz and Schreiber 2004].

\subsubsection{O Método de Rosenstein - Small Data Sets}

Esse método pode ser esquematizado da seguinte maneira:

1) Calcula-se o período médio $P$ da série temporal;

2) Calcula-se a dimensão de correlacão, e a dimensão de incorporação através do Teorema de Takens, $\left(m \geq 2 D_{2}+1\right)$ [Takens 1981];

3) Reconstrui-se o espaço de fase;

4) Encontram-se os vizinhos mais próximos na trajetória:

$$
d_{j}(0)=\min _{x_{j}}\left\|\mathbf{X}_{i}-\mathbf{X}_{j}\right\|, \quad|i-j|>\mathbf{P}
$$

5) Os j-ésimos vizinhos mais próximos divergem a uma taxa aproximadamente igual ao expoente de Lyapunov:

$$
d_{j}(i) \approx d_{j}(0) e^{\lambda_{1} i}
$$

Extraindo do logaritmo de ambos os lados temos:

$$
\ln d_{j}(i) \approx \ln d_{j}(0)+\lambda_{1} i
$$

6) Estima-se o expoente de Lyapunov com o uso do método dos mínimos quadrados:

$$
y(i)=\frac{1}{\Delta t}\left\langle\ln d_{j}(i)\right\rangle
$$

na qual $\langle\ldots\rangle$ denota a média de todos os valores de $j$ [Feng et al. 2009], [Rosenstein et al. 1993]. Um algoritmo de MATLAB para este cálculo está disponível em [mirwais 2020].

\subsection{O Parâmetro de Hurst}

O parâmetro de Hurst, primeiro formulado em [Hurst 1956], é usado para classificar séries temporais quanto ao seu comportamento de persistência ou memória:

$H<0,5$ - as medidas tendem a alternar entre valores baixos e valores altos (periodicidade);

$H \approx 0,5$ - as medidas tendem a apresentar valores com uma distribuição estocástica (aleatória);

$H>0,5$ - valores altos tendem a ser seguidos por outros valores altos; valores baixos tendem a ser seguidos por valores baixos e essa tendência se mantêm num futuro infinito (persistência de longa duração) [Hurst 1956], [Mandelbrot 1968].

\section{Proposta de Predição de Tráfego}

A predição do tráfego adotada é baseada no método DRA (Dynamic Rate Allocation, Figura 1) e no método descrito em [Liu and Zhang 2016]. Fundamentados em 
([Leland et al. 1994], [Trinh and Miki 1999] e [Rocha and Teles 2013] definem a variável processo de tráfego de dados médios, dividindo o tráfego em blocos de tamanho $b$ :

$$
Y_{n}^{(b)}=\frac{1}{b}\left(Y_{n b-b+1}+\ldots+Y_{n b}\right)
$$

na qual $Y$ é o espaço de fase reconstruído, $\mathrm{b}, \mathrm{n}=1,2, \ldots, b$ é o tamanho do bloco e $n$ é o número do bloco.

No método $D R A$ (Figura 1), o preditor para $Y_{n}{ }^{(b)}$ pode ser obtido da seguinte maneira:

$$
\hat{Y}_{n}^{(b)}=a Y_{n-1}{ }^{(b)}+(1-a) \mu,
$$

na qual $a=\left(2^{2 H}-2\right) / 2, a \in(0,1)$, e $H$ é o parâmetro de Hurst.

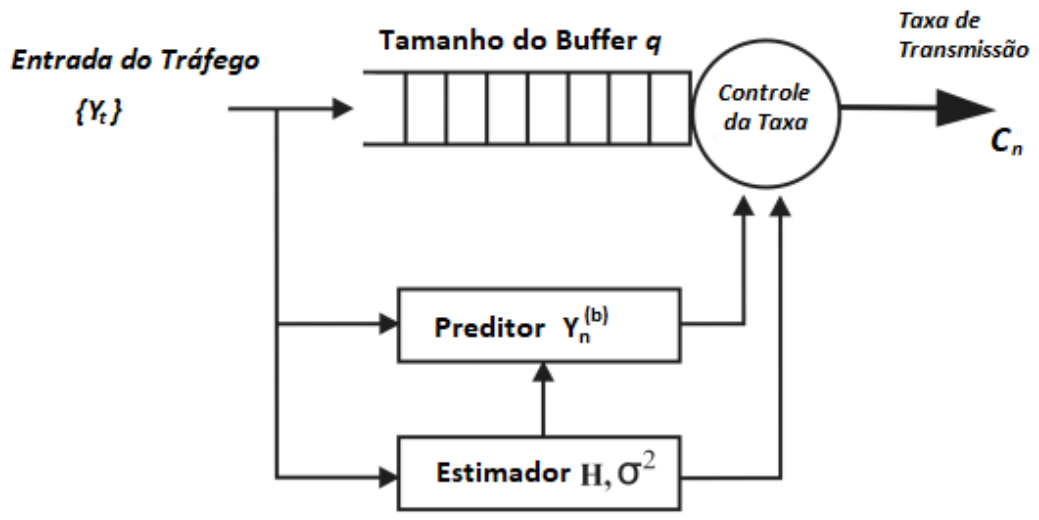

Figura 1. Esquema da alocação dinâmica de recursos [Rocha and Teles 2013]

No método proposto, a predição de tráfego, através do expoente de Lyapunov, é descrita em seguida, com base em [Rosenstein et al. 1993].

Seja $Y_{j}$, no espaço de fase, o ponto central previsto, e $Y_{k}$ o ponto mais próximo dele com a distância $d_{j}(0)$ de $Y_{j}$, que é a menor das distâncias entre todos os pontos do atrator. Assim, existe uma relação que segue a seguinte estrutura:

$$
d_{j}(0)=\left\|Y_{j}-Y_{k}\right\| \text {. }
$$

No próximo passo, $Y_{j}$ e $Y_{k}$ evoluem para $Y_{j+1}$ e $Y_{k+1}$ respectivamente. Se o expoente de Lyapunov mede a taxa de expansão das órbitas ao longo do tempo, e o parâmetro de Hurst reflete as correlações temporais entre as amostras, ajustam-se as amostras da previsão para que possuam a mesma correlação do tráfego original:

$$
\left\|Y_{j+1}-Y_{k+1}\right\|=\left\{\left\|Y_{j}-Y_{k}\right\| * e^{\lambda_{1}}\right\}^{H}=\left\{d(0) * e^{\lambda_{1}}\right\}^{H} .
$$

O único ponto desconhecido será $x\left(t_{n+1}\right)$, o último componente do ponto de fase $Y_{j+1}$, e poderá ser obtido a partir da seguinte Equação:

$$
Y_{j+1}(m)=Y_{k+1}(m) \pm \sqrt{\left(\left(d_{j}(0) e^{\lambda_{1}}\right)^{H}\right)^{2}-\sum_{i=1}^{m-1}\left[Y_{j+1}(i)-Y_{k+1}(i)\right]^{2}} .
$$


O valor previsto $\hat{x}\left(t_{n+1}\right)$, usado como valor conhecido no vetor posterior, pode ser obtido da seguinte maneira:

$$
\hat{x}\left(t_{n+1}\right)=Y_{j+1}(m) .
$$

Em ambos métodos, alocação de taxa é realizada em seguida a fim de garantir o funcionamento da rede dinâmica, cujo processo é discutido na próxima Seção.

\section{Alocação Dinâmica de Taxa}

A taxa de alocação $C$ para o tráfego previsto $Y_{n}{ }^{(b)}$ na Seção anterior é encontrada da seguinte maneira [Rosenstein et al. 1993], [Rocha and Teles 2013]:

$$
C_{n}=Y_{n}^{(b)}+\Delta C,
$$

para um $\Delta C$ encontrado da seguinte maneira:

$$
\Delta C \approx\left\{\frac{-2 \ln \epsilon+2 \ln \left(\frac{1}{\sqrt{2 \pi}}\right) y^{-1} \sigma(1-a) H^{H}(1-H)^{1-H}}{q^{1-H}}\right\}^{1 / H}
$$

na qual $\epsilon$ é a probabilidade de transbordo do buffer, $\sigma$ é o desvio padrão do tráfego correspondente e $q$ é a capacidade do buffer. A variável $y$ está relacionada da seguinte maneira:

$$
\epsilon \approx \frac{1}{\sqrt{2 \pi}} y^{-1} \exp \left(-\frac{1}{2} y^{2}\right)
$$

\section{Experimentos de Análise das Séries Temporais}

Os resultados experimentais encontrados nas análises das séries reais de tráfego de rede estão descritos à seguir, e os intervalos utilizados nessa análise, para cada série, estão organizados na Tabela 1. As 5000 amostras de cada série estão ilustradas na Figura 2.

\begin{tabular}{|l|c|r|c|c|r|r|r|c|c|}
\cline { 2 - 10 } \multicolumn{1}{c|}{} & intervalo & iterações & $\tau$ & $D_{2}$ & $\mathrm{~m}$ & tlinear & fs & $\lambda$ & H \\
\hline BCpAug89 & $25000: 30000$ & 100 & 1 & 0,22 & 2 & $1: 2$ & 1 & 0,9753 & 0,6820 \\
\hline dec-pkt-2 & $25000: 30000$ & 100 & 1 & 2,09 & 6 & $1: 6$ & 1 & 0,3824 & 0,6893 \\
\hline MultipathTCP & $25000: 30000$ & 100 & 1 & 0,58 & 3 & $1: 3$ & 0,1 & 0,0090 & 0,5685 \\
\hline
\end{tabular}

Tabela 1. Parâmetros do Método de Rosenstein, Expoente de Lyapunov $\lambda$ e Parâmetro de Hurst $H$

As séries BCpAug89 e dec-pkt-2 são de tráfego Ethernet (da Bellcore Morristown Research and Engineering [Danzig et al. 2000]) e a série MultipathTCP representa o tráfego de uma rede sem fio (wireless) que utiliza o protocolo Multipath TCP ([Coninck et al. 2016]), esse, por sua vez, uma extenção do protocolo de controle de transmissão ( TCP - Transmission Control Protocol). Estas séries foram escolhidas por estarem amplamente presentes na literatura ([Rocha 2016], [Silva 2019]), possibilitando a comparação entre resultados [Tian 2020]. 


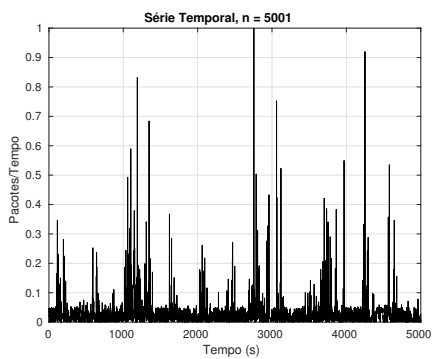

(a) BCpAug89

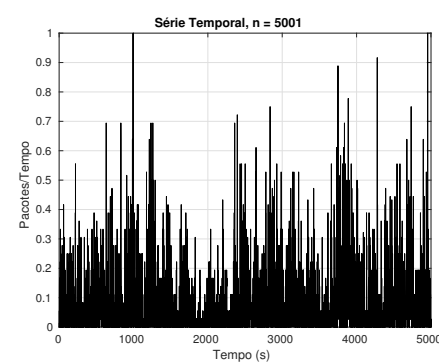

(b) dec-pkt-2

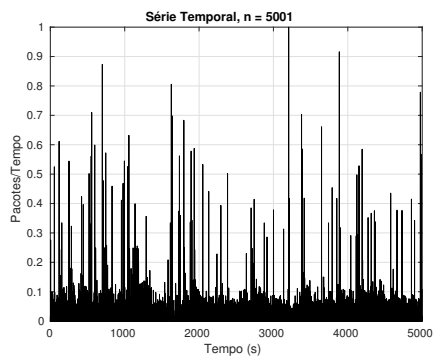

(c) MultipathTCP

Figura 2. Séries Temporais $\mathrm{N}=5001$

Ficam evidentes nos gráficos das três séries a presença de rajadas, cujas amplitudes são bastante elevadas em relação à media, principalmente para as séries BCpAug89 (Figura 2a) e MultipathTCP (Figura 2c).

Os gráficos das integrais de correlação (Figura 3) para espaços de fase reconstruídos ([Grassberger and Procaccia 1983a], [Grassberger and Procaccia 1983b]), apresentam as retas referentes a valores da dimensão de incorporação variando entre $m=2 \mathrm{e}$ $m=16$, de cima para baixo nas retas dos gráficos [Feng et al. 2009].

A relação entre $C_{m}(r)$ e $r$ indica regiões de escala, nas séries da Figura 3a e 3b, para todas as dimensões de incorporação usadas, evidenciadas no afunilamento das retas, sinal de uma lei de potência (power law) presente, permitindo assim estimativas relativamente precisas das dimensões de correlação $D_{2}$ [Kantz and Schreiber 2004]. A série da Figura $3 \mathrm{c}$ apresenta retas quase paralelas, indicando ausência de uma lei de potência.

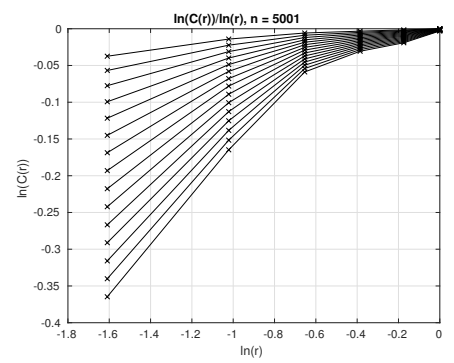

(a) BCpAug89

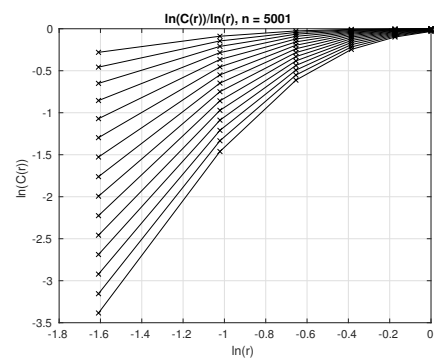

(b) dec-pkt-2

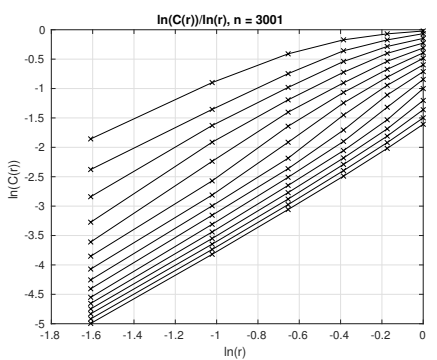

(c) MultipathTCP

Figura 3. Integrais de Correlação, $\mathrm{N}=5001$

A Figura 4 mostra a dimensão de correlação $D_{2}$ em relação à dimensão de incorporação $m$, e os valores de $\tau$ e $D_{2}$ encontrados para essas séries estão organizados na Tabela 1, na qual se observa que a série dec-pkt-2 possui maior dimensionalidade entre todas as séries analisadas.

Nos gráficos da Figura 5 pode-se observar a faixa de valores necessária para o cálculo do expoente de Lyapunov, organizados na Tabela 1, juntamente com todos os $\lambda$ calculados para cada série, pois este está associado à inclinação da reta em sua parcela linear [Feng et al. 2009]. Por exemplo, ela mostra que para a Figura 5a, o intervalo necessário para o cálculo é aquele de 1 até 2 (ou seja, as primeiras 2 iterações no eixo iterações) para uma frequência de amostragem $f s=1$. 


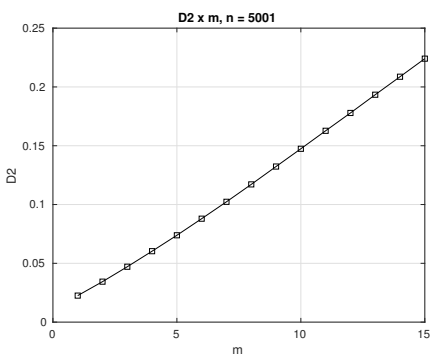

(a) BCpAug89

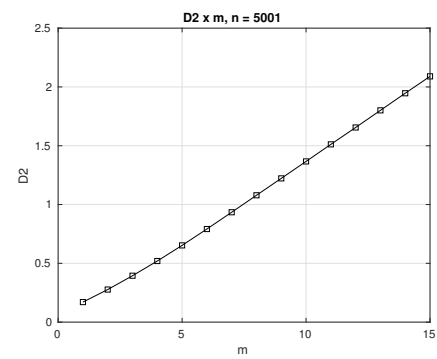

(b) dec-pkt-2

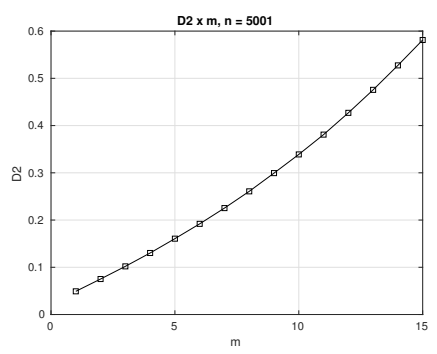

(c) MultipathTCP

Figura 4. $\mathrm{D} 2 \times \mathrm{m}, \mathrm{N}=5001$

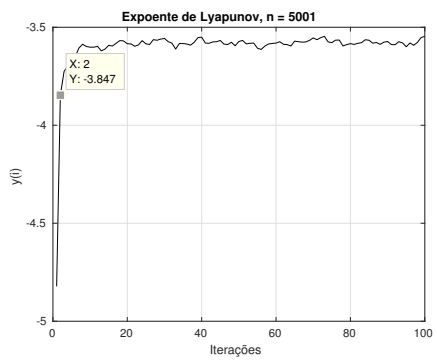

(a) BCpAug89

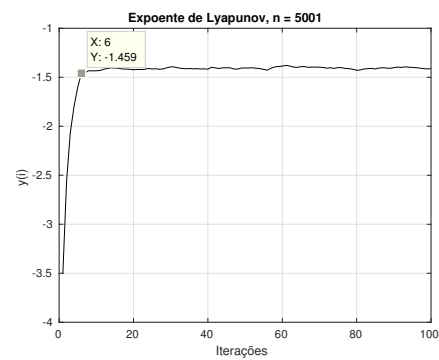

(b) dec-pkt-2

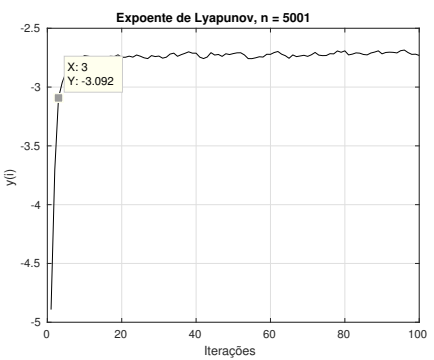

(c) MultipathTCP

Figura 5. $y(t) \mathbf{x} i \Delta t, \mathbf{n}=\mathbf{5 0 0 1}$

O parâmetro de Hurst foi calculado através do método descrito em [Rocha and Teles 2013]. Como mostrado na Tabela 1, as séries BCpAug89 e dec-pkt-2 apresentam dependência de longa duração, característica de séries caóticas autossimilares $(0,5<H<1)$. A série MultipathTCP apresenta aleatoriedade, com um $H \approx 0,5$.

A previsão das séries temporais por meio de $\lambda$ e $H$ está ilustrada na Figura 6, na qual pode-se observar que a acurácia do algoritmo proposto foi bastante satisfatória (baixos valores de erro médio quadrático) para a previsão e a alocação de taxa, cujos valores estão organizados na Tabela 2.

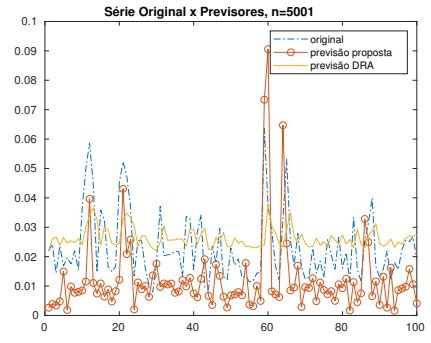

(a) BCpAug89

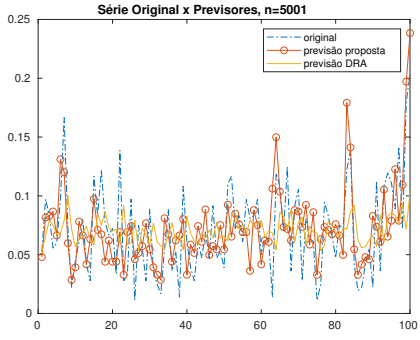

(b) dec-pkt-2

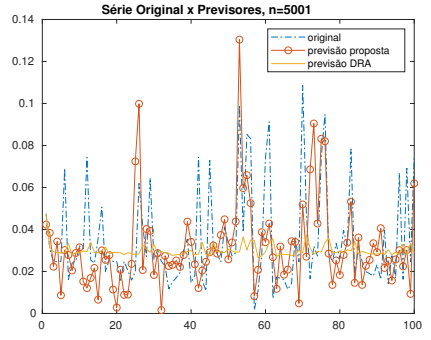

(c) MultipathTCP

Figura 6. Previsão Proposta $x$ Previsor do Método DRA, N=5001

Para a dec-pkt-2 e a MultipathTCP o método proposto teve desempenho mais preciso quando comparado ao método $D R A$, e para a série $\mathrm{BCpAug89,} \mathrm{o} \mathrm{método} D R A$ obteve um desempenho ligeiramente superior ao do método proposto.

A alocação dinâmica de taxa está ilustrada na Figura 7. Nela observa-se que esse processo seguiu o desempenho da previsão, de forma que o método proposto apresentou 


\begin{tabular}{|l|r|r|r|r|}
\cline { 2 - 5 } \multicolumn{1}{c|}{} & \multicolumn{2}{c|}{ método proposto } & \multicolumn{2}{c|}{ método $D R A$} \\
\cline { 2 - 5 } \multicolumn{1}{c|}{ MSE } & previsão & alocação de taxa & previsão & alocação de taxa \\
\hline BCpAug89 & 0,0002 & 0,0002 & 0,0001 & 0,0001 \\
\hline dec-pkt-2 & 0,0008 & 0,0008 & 0,0014 & 0,0014 \\
\hline MultipathTCP & 0,0004 & 0,0004 & 0,0005 & 0,0005 \\
\hline
\end{tabular}

Tabela 2. Erro Médio Quadrático (MSE)

desempenho mais preciso para as séries dec-pkt-2 e MultipathTCP, enquanto que, para a série BCpAug89, o desempenho ficou ligeiramente inferior ao do método $D R A$.

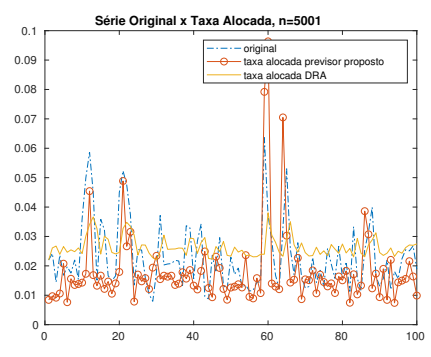

(a) BCpAug89

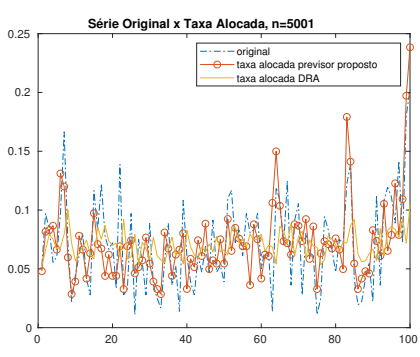

(b) dec-pkt-2

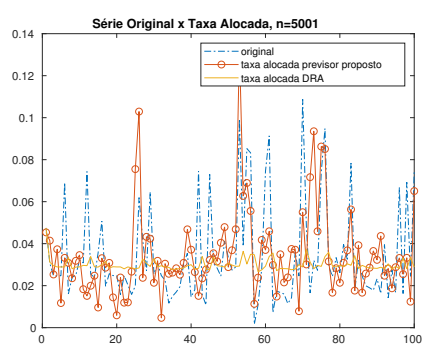

(c) MultipathTCP

Figura 7. Taxa Alocada Proposta x Taxa Alocada Método DRA, N=5001

\section{Conclusão}

Nesse trabalho, o objetivo foi examinar o desempenho de algoritmos que otimizam o cálculo de parâmetros de quantificação dos sistemas caóticos, analisando os valores obtidos de forma a ponderar suas acurácias, para então simular uma previsão temporal e alocação dinâmica de taxa de serviço para os servidores de rede. Foram analisadas séries temporais de tráfego de redes sob a perspectiva da dinâmica não-linear, através da qual foi possível estimar a dimensão de correlação com o uso do algoritmo de GrassbergerProcaccia, a qual apontou um valor fractal (fracionário). O algoritmo Small Sets foi usado para calcular o maior expoente de Lyapunov, que retornou um valor positivo, indicando órbitas expansivas. O parâmetro de Hurst confirmou a presença de dependência de longa duração entre as amostras para duas das séries analisadas. Uma delas, a InterMultipathTCP, apresentou um $H \approx 0,5$ mas um $m$ bastante baixo, evidenciando um comportamento misto entre caos (baixa dimensionalidade) e aleatoriedade $(H \approx 0,5)$.

De modo geral, todos os métodos utilizados no estudo confirmaram características esperadas para uma distribuição caótica, e os métodos de previsão e alocação de taxa foram eficientes na predição e alocação de taxa para todas as séries analisadas, com valores de $M S E$ bastante reduzidos. Para tais valores reduzidos de erro médio quadrático, ou seja, uma maior similaridade entre a série real e a taxa de transmissão, são esperados também menores valores para taxas de transbordo de buffer, com uma menor ocupação deste, de forma que a utilização do enlace de transmissão seja otimizada. Para trabalhos futuros, pretende-se aprofundar as análises referentes à previsão e alocação dinâmica de taxa de serviço, atentando para métricas como taxa de transbordo do buffer e probabilidade de perdas. 


\section{Referências}

Alves, P. (2019). Chaos in historical prices and volatilities with five-dimensional euclidean spaces. Chaos, Solitons \& Fractals: X, 1:100002.

Alves, P., Duarte, L., and [da Mota], L. (2017). A new characterization of chaos from a time series. Chaos, Solitons \& Fractals, 104:323 - 326.

Alves, P., Duarte, L., and [da Mota], L. (2018). Detecting chaos and predicting in dow jones index. Chaos, Solitons \& Fractals, 110:232 - 238.

Berge, P., Pomeau, Y., and Vidal, C. (1987). Order Within Chaos. Wiley.

Coninck, Q. D., Baerts, M., Hesmans, B., and Bonaventure, O. (2016). CRAWDAD dataset uclouvain/mptcp_smartphone (v. 2016-03-04). Downloaded from https : / / crawdad.org/uclouvain/mptcp_smartphone/20160304.

Danzig, P., Mogul, J., Paxson, V., and Schwartz, M. (2000). The internet traffic archive datasets. Downloaded from http://ita.ee.lbl.gov/. traceset: combined. Último acesso em 09-22-2010.

Feng, H., Shu, Y., and Yang, O. W. (2009). Nonlinear analysis of wireless lan traffic. Nonlinear Analysis: Real World Applications, 10(2):1021 - 1028.

Fraser, A. M. and Swinney, H. L. (1986). Independent coordinates for strange attractors from mutual information. Phys. Rev. A, 33:1134-1140.

Gleick, J. (1987). Chaos: Making a New Science. Penguin Books, USA.

Grassberger, P. and Procaccia, I. (1983a). Characterization of strange attractors.

Grassberger, P. and Procaccia, I. (1983b). Measuring the strangeness of strange attractors. Physica D, 9:189.

Hurst, H. E. (1956). The problem of long-term storage in reservoirs. Hydrological Sciences Journal, 1(3):13-27.

Kantz, H. and Schreiber, T. (2004). Nonlinear time series analysis, volume 7. Cambridge university press.

Kaplan, D. T. and Glass, L. (1993). Coarse-grained embeddings of time series: random walks, gaussian random processes, and deterministic chaos. Physica D: Nonlinear Phenomena, 64(4):431-454.

Leland, W. E., Taqqu, M. S., Willinger, W., and Wilson, D. V. (1994). On the self-similar nature of ethernet traffic (extended version). IEEE/ACM Transactions on Networking, 2(1):1-15.

Liebert, W. and Schuster, H. G. (1989). Proper choice of the time delay for the analysis of chaotic time series. Physics Letters A, 142(2-3):107-111.

Liu, Y. and Zhang, J. (2016). Predicting traffic flow in local area networks by the largest lyapunov exponent. Entropy, 18(1):32.

Lorenz, E. (1963). Deterministic nonperiodic flow. Journal of Atmospheric Sciences, 20(2):130-148.

Lorenz, E. (1995). The Essence Of Chaos. Jessie and John Danz lectures. Taylor \& Francis. 
Mandelbrot, B. B. (1968). Noah, joseph, and operational hydrology. Water resources research. 4 (5) : 909-918; 1968.

Mandelbrot, B. B. (1997). The variation of certain speculative prices. In Fractals and scaling in finance, pages 371-418. Springer.

Mandelbrot, B. B. and Hudson, R. L. (2004). The misbehaviour of markets: a fractal view of risk, ruin and reward. Profile, London, UK.

Mañé, R. (1981). On the dimension of the compact invariant sets of certain non-linear maps. Lecture Notes in Mathematics, Berlin Springer Verlag, 898:230.

mirwais (2020). Largest lyapunov exponent with rosenstein's algorithm. MATLAB Central File Exchange. Retrieved February 13, 2020.

Prigogine, I. and Stengers, I. (1984). Order Out of Chaos Man's New Dialogue with Nature /Ilya Prigogine and Isabelle Stengers ; Foreword by Alvin Toffler. -. -. Bantam Books, 1984.

Rocha, F. G. C. (2016). Alocação de recursos em redes sem fio ofdm multiusuário utilizando modelagem multifractal adaptativa.

Rocha, F. G. C. and Teles, F. H. V. (2013). Adaptive rate control based on loss probability estimation considering a cascade based multifractal model for the network traffic. IJWMIP, 11.

Rosenstein, M. T., Collins, J. J., and Luca, C. J. D. (1993). A practical method for calculating largest lyapunov exponents from small data sets. Physica D: Nonlinear Phenomena, 65(1):117 - 134.

Silva, M. R. P. d. (2019). Propostas de um modelo para o tráfego de redes de comunicações e de funções de janela para o filtro fir utilizado na tecnologia f-ofdm.

Solis, P. (2007). Uma metodologia de engenharia de tráfego baseada na abordagem autosimilar para a caracterização de parâmetros e a otimização de redes multimídia.

Strogatz, S. (2012). Sync: How Order Emerges From Chaos In the Universe, Nature, and Daily Life. Hyperion.

Strogatz, S. H. (2015). Nonlinear dynamics and chaos: with applications to physics, biology, chemistry, and engineering. CRC Press.

Takens, F. (1981). Detecting strange attractors in turbulence. Lecture Notes in Mathematics, Berlin Springer Verlag, 898:366.

Tempelman, J. R. and Khasawneh, F. A. (2020). A look into chaos detection through topological data analysis. Physica D: Nonlinear Phenomena, 406:132446.

Tian, Z. (2020). Chaotic characteristic analysis of network traffic time series at different time scales. Chaos, Solitons \& Fractals, 130:109412.

Trinh, N. C. and Miki, T. (1999). Dynamic resource allocation for self-similar traffic in atm network. Fifth Asia-Pacific Conference on ... and Fourth Optoelectronics and Communications Conference on Communications, , 1:160-165 vol.1.

Wolf, A., Swift, J. B., Swinney, H. L., and Vastano, J. A. (1985). Determining lyapunov exponents from a time series. Physica D: Nonlinear Phenomena, 16(3):285 - 317. 